\title{
Monte Carlo simulations and $n-p$ differential scattering data measured with Proton Recoil Telescopes
}

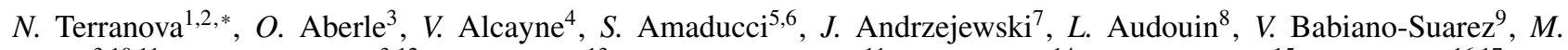

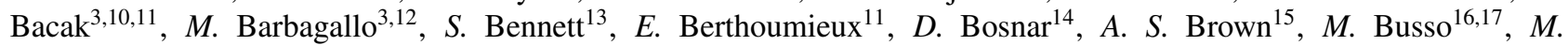
Caamaño $^{18}, L$. Caballero ${ }^{9}, M$. Calviani ${ }^{3}, F$. Calviño ${ }^{19}, D$. Cano-Ott $^{4}, A$. Casanovas ${ }^{19}, F$. Cerutti $^{3}, E$. Chiaveri $^{13,20,3}, N$. Colonna $^{12}$, G. P. Cortés ${ }^{19}$, M. A. Cortés-Giraldo ${ }^{20}, L$. Cosentino $^{5}, S$. Cristallo ${ }^{16,21}$, L. A. Damone ${ }^{12,22}, P . J$. Davies $^{13}$, M. Diakaki ${ }^{23}, M$. Dietz ${ }^{24}, C$. Domingo-Pardo ${ }^{9}, R$. Dressler ${ }^{25}, Q$. Ducasse $^{26}, E$. Dupont ${ }^{11}, I$. Durán ${ }^{18}, Z$. Eleme ${ }^{27}$, $B$. Fernández-Domíngez ${ }^{18}, A$. Ferrari ${ }^{3}, I$. Ferro-Gonçalves ${ }^{28}, P$. Finocchiaro ${ }^{5}, V$. Furman ${ }^{29}, R$. Garg ${ }^{24}, A$. Gawlik ${ }^{7}, S$. Gilardoni $^{3}, K$. Göbel ${ }^{30}, E$. González-Romero ${ }^{4}, C$. Guerrero ${ }^{20}, F$. Gunsing ${ }^{11}, S$. Heinitz ${ }^{25}, J$. Heyse ${ }^{31}, D$. G. Jenkins $^{15}, E$.

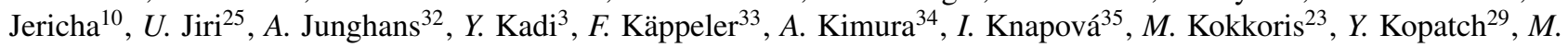
$\mathrm{Krtička}^{35}, D$. Kurtulgil ${ }^{30}, I$. Ladarescu ${ }^{9}, C$. Lederer-Woods ${ }^{24}, J$. Lerendegui-Marco ${ }^{20}$, S.-J. Lonsdale ${ }^{24}, D$. Macina $^{3}, A$.


E. Mendoza ${ }^{4}, A$. Mengoni ${ }^{36,40}, V$. Michalopoulou ${ }^{3,23}, P . M$. Milazzo $^{41}, M$. A. Millán-Callado ${ }^{20}, F$. Mingrone ${ }^{3}, J$. MorenoSoto $^{11}, A$. Musumarra ${ }^{5,6}, A$. Negret ${ }^{42}, F$. Ogállar ${ }^{43}, A$. Oprea $^{42}, N$. Patronis ${ }^{27}, A$. Pavlik ${ }^{44}, J$. Perkowski ${ }^{7}, C$. Petrone ${ }^{42}, L$. Piersanti $^{16,21}, E$. Pirovano ${ }^{26}, I$. Porras ${ }^{43}, J$. Praena ${ }^{43}, J . M$. Quesada ${ }^{20}, D$. Ramos Doval $^{8}, R$. Reifarth ${ }^{30}, D$. Rochman $^{25}, C$. Rubbia $^{3}, M$. Sabaté-Gilarte ${ }^{20,3}, A$. Saxena $^{45}, P$. Schillebeeckx $^{31}, D$. Schumann $^{25}, A$. Sekhar $^{13}, A . G$. Smith $^{13}, N$. Sosnin $^{13}$, P. Sprung ${ }^{25}, A$. Stamatopoulos ${ }^{23}, G$. Tagliente ${ }^{12}, J$. L. Tain ${ }^{9}, A$. E. Tarifeño-Saldivia ${ }^{19}, L$. Tassan-Got ${ }^{3,23,8}, B$. Thomas ${ }^{30}$, $P$. Torres-Sánchez ${ }^{43}, A$. Tsinganis ${ }^{3}, S$. Urlass ${ }^{3,32}, S$. Valenta ${ }^{35}, G$. Vannini ${ }^{36,37}, V$. Variale ${ }^{12}, P$. Vaz $^{28}, A$. Ventura ${ }^{2}, D$.

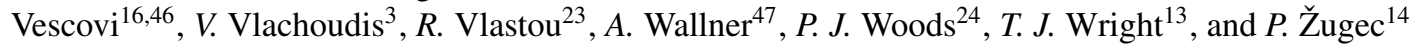

${ }^{1}$ Agenzia nazionale per le nuove tecnologie, l'energia e lo sviluppo economico sostenibile (ENEA), Frascati, Italy

${ }^{2}$ Istituto Nazionale di Fisica Nucleare, CNAF, Bologna, Italy

${ }^{3}$ European Organization for Nuclear Research (CERN), Switzerland

${ }^{4}$ Centro de Investigaciones Energéticas Medioambientales y Tecnológicas (CIEMAT),Spain

${ }^{5}$ INFN Laboratori Nazionali del Sud, Catania, Italy

${ }^{6}$ Dipartimento di Fisica e Astronomia, Università di Catania, Italy

${ }^{7}$ University of Lodz, Poland

${ }^{8}$ IPN, CNRS-IN2P3, Univ. Paris-Sud, Université Paris-Saclay, F-91406 Orsay Cedex,France

${ }^{9}$ Instituto de Física Corpuscular, CSIC - Universidad de Valencia, Spain

${ }^{10}$ Technische Universität Wien, Austria

${ }^{11}$ CEA Saclay, Irfu, Université Paris-Saclay, Gif-sur-Yvette, France

${ }^{12}$ Istituto Nazionale di Fisica Nucleare, Bari, Italy

${ }^{13}$ University of Manchester, United Kingdom

${ }^{14}$ Department of Physics, Faculty of Science, University of Zagreb, Croatia

${ }^{15}$ University of York, United Kingdom

${ }^{16}$ Istituto Nazionale di Fisica Nazionale, Perugia, Italy

${ }^{17}$ Dipartimento di Fisica e Geologia, Università di Perugia, Italy

${ }^{18}$ University of Santiago de Compostela, Spain

${ }^{19}$ Universitat Politècnica de Catalunya, Spain

${ }^{20}$ Universidad de Sevilla, Spain

${ }^{21}$ Istituto Nazionale di Astrofisica - Osservatorio Astronomico d'Abruzzo, Italy

${ }^{22}$ Dipartimento di Fisica, Università degli Studi di Bari, Italy

${ }^{23}$ National Technical University of Athens, Greece

${ }^{24}$ School of Physics and Astronomy, University of Edinburgh, United Kingdom

${ }^{25}$ Paul Scherrer Institut (PSI), Villigen, Switzerland

${ }^{26}$ Physikalisch-Technische Bundesanstalt (PTB), Bundesallee 100, 38116 Braunschweig, Germany

${ }^{27}$ University of Ioannina, Greece

${ }^{28}$ Instituto Superior Técnico, Lisbon, Portugal

${ }^{29}$ Joint Institute for Nuclear Research (JINR), Dubna, Russia

${ }^{30}$ Goethe University Frankfurt, Germany

${ }^{31}$ European Commission, Joint Research Centre, Geel, Retieseweg 111, B-2440 Geel,Belgium

${ }^{32}$ Helmholtz-Zentrum Dresden-Rossendorf, Germany

${ }^{33}$ Karlsruhe Institute of Technology, Campus North, IKP, 76021 Karlsruhe, Germany

${ }^{34}$ Japan Atomic Energy Agency (JAEA), Tokai-mura, Japan 


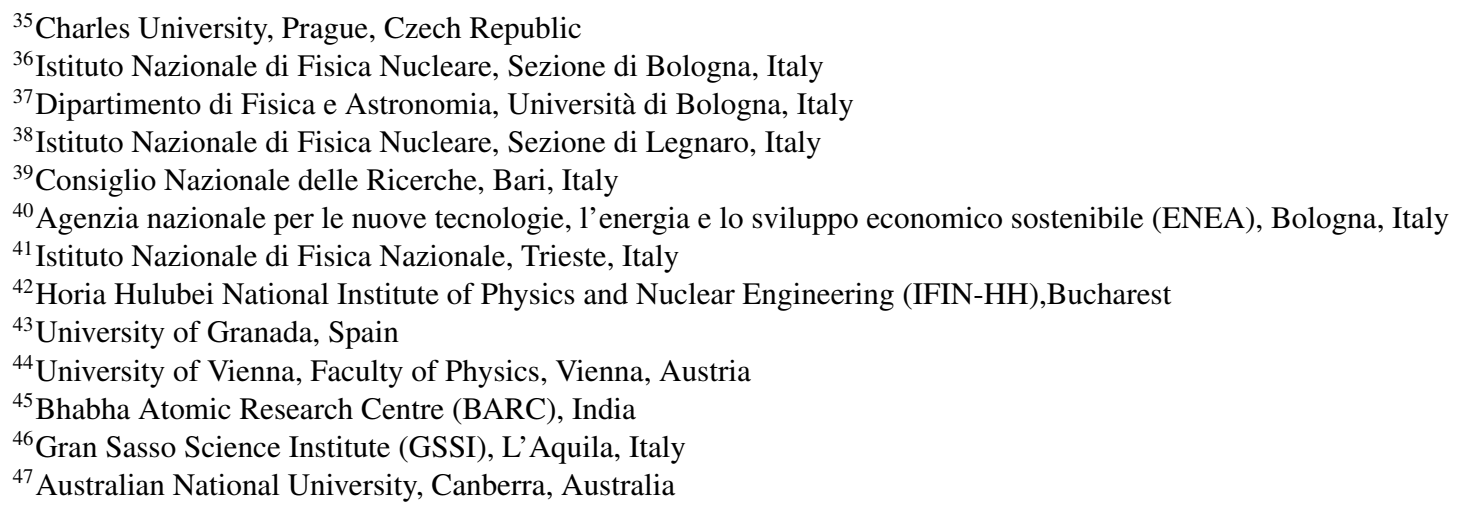

\begin{abstract}
The neutron-induced fission cross section of ${ }^{235} \mathrm{U}$, a standard at thermal energy and between 0.15 $\mathrm{MeV}$ and $200 \mathrm{MeV}$, plays a crucial role in nuclear technology applications. The long-standing need of improving cross section data above $20 \mathrm{MeV}$ and the lack of experimental data above $200 \mathrm{MeV}$ motivated a new experimental campaign at the $\mathrm{n}_{-}$TOF facility at CERN. The measurement has been performed in 2018 at the experimental area 1 (EAR1), located at $185 \mathrm{~m}$ from the neutron-producing target (the experiment is presented by A. Manna et al. in a contribution to this conference). The ${ }^{235} \mathrm{U}(\mathrm{n}, \mathrm{f})$ cross section from $20 \mathrm{MeV}$ up to about $1 \mathrm{GeV}$ has been measured relative to the ${ }^{1} \mathrm{H}(\mathrm{n}, \mathrm{n}){ }^{1} \mathrm{H}$ reaction, which is considered the primary reference in this energy region. The neutron flux impinging on the ${ }^{235} \mathrm{U}$ sample (a key quantity for determining the fission events) has been obtained by detecting recoil protons originating from n-p scattering in a $\mathrm{C}_{2} \mathrm{H}_{4}$ sample. Two Proton Recoil Telescopes (PRT), consisting of several layers of solid-state detectors and fast plastic scintillators, have been located at proton scattering angles of $25.07^{\circ}$ and $20.32^{\circ}$, out of the neutron beam. The PRTs exploit the $\Delta \mathrm{E}-\mathrm{E}$ technique for particle identification, a basic requirement for the rejection of charged particles from neutron-induced reactions in carbon. Extensive Monte Carlo simulations were performed to characterize proton transport through the different slabs of silicon and scintillation detectors, to optimize the experimental set-up and to deduce the efficiency of the whole PRT detector. In this work we compare measured data collected with the PRTs with a full Monte Carlo simulation based on the Geant-4 toolkit.
\end{abstract}

\section{Introduction}

The ${ }^{235} \mathrm{U}(\mathrm{n}, \mathrm{f})$ cross section is considered as standard at thermal neutron energy and between $0.15 \mathrm{MeV}$ and 200 $\mathrm{MeV}$ [1]. Its importance in nuclear reactor applications is overwhelming and, typically, it is employed as a reference in fission cross section measurements, see Ref. [2] among the others. Despite its widespread use in many fields, only two measurements are available between 20 and $200 \mathrm{MeV}[3,4]$, and no experimental points exist for neutron-induced fission cross sections above $200 \mathrm{MeV}$. At the neutron time-of-flight facility n_TOF [5], via an INFNPTB (Istituto Nazionale di Fisica Nucleare, PhysikalischTechnische Bundesanstalt) joint experimental campaign, the ${ }^{235} \mathrm{U}(\mathrm{n}, \mathrm{f})$ cross section from $20 \mathrm{MeV}$ up to about $1 \mathrm{GeV}$ has been measured relative to the ${ }^{1} \mathrm{H}(\mathrm{n}, \mathrm{n}){ }^{1} \mathrm{H}$ reaction [6]. Fission events form several ${ }^{235} \mathrm{U}$ samples have been detected using fission chambers and a set of parallel plate avalanche counters (PPAC) [6]. To measure n-p scattering in presence of an intense $\gamma$ flash and a continuous neutron energy distribution, three Proton Recoil Telescopes (PRTs) were specifically designed. Since fission fragments and recoil protons have been measured by different experimental set-ups, efficiencies must be precisely known and a fully characterization of the detectors is mandatory. Here, we present the model used to simulate the PRT systems employing the GEANT4 toolkit [7]. Some preliminary results and comparisons to experimental data for the two of the three PRT detectors of INFN conception are also shown.

*e-mail: nicholas.terranova@enea.it

\section{Detection system overview}

To measure n-p scattering, three PRT detection systems were placed in front of Polyethylene targets as shown in figure 1 . In order to cover a broader neutron energy do-

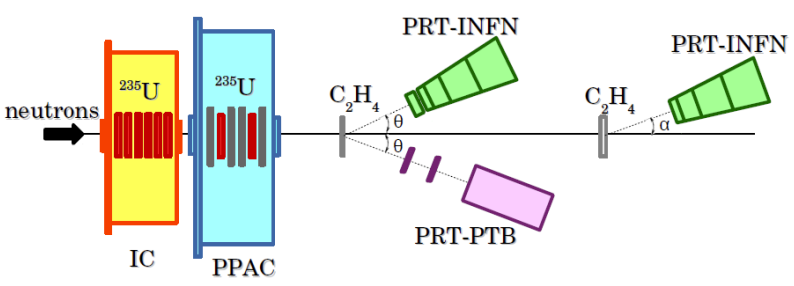

Figure 1. Experimental set-up for the ${ }^{235} \mathrm{U}(\mathrm{n}, \mathrm{f})$ cross section relative to ${ }^{1} \mathrm{H}(\mathrm{n}, \mathrm{n}){ }^{1} \mathrm{H}$.

main, the design proposed in Ref. [8] was extended to multi-stage systems with increased particle discrimination potential. Two INFN-PRTs consisting of several layers of solid-state detectors and fast plastic scintillators were designed to cover the whole neutron energy range from 20 $\mathrm{MeV}$ to $1 \mathrm{GeV}$. The former PRT, called hereinafter INFNPRT-L, was foreseen for lower energies (from 20 to about $200 \mathrm{MeV}$ ), being equipped with two frontal solid-state layers preceding 4 stages of plastic scintillation material. The latter (INFN-PRT-H) is made only of multiple scintillation stages, since thin solid-state layers would not contribute to any detection at higher energies. Both were located in front of Polyethylene targets of different thicknesses, 
at proton scattering angles of $25.07^{\circ}$ and $20.32^{\circ}$, respectively, out of the neutron beam. The $\Delta \mathrm{E}-\mathrm{E}$ technique has been used to perform particle identification and subtract the background both from secondaries produced by reactions on Carbon nuclei in the Polyethylene target, and from spourious energy deposits due to undesired particles hitting the detector sensitive material.

\section{Monte Carlo simulation development}

The evaluation of the n-p scattering obtained by Arndt and his collaborators $[9,10]$ was accepted by the NEANDC/INDC as a primary standard for cross section measurements in the 20-350 MeV range [11]. In Geant4, the application developer is called to directly define the physics of his own problem adopting the physical models made available in the toolkit. To obviate such a complexity, reference physics list classes are already defined by Geant 4 developers to be easily included by users for implementing new applications. Unfortunately, no reference physics list involves Arndt data. In figure 2 several

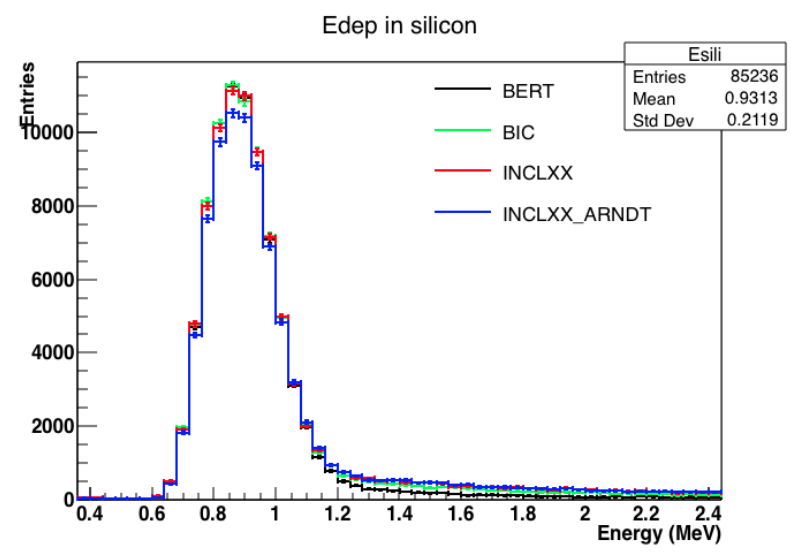

Figure 2. Comparison between different physics lists in Geant4 for energy deposition in the first solid-state layer of the PRT detector for $50 \mathrm{MeV}$ neutrons impinging on the target.

reference physics lists which are available in Geant4 have been compared to a user-defined physics class including Arndt evaluation. A simplified $\Delta \mathrm{E}-\mathrm{E}$ detector made of one solid-state layer coupled to a plastic scintillator was chosen to perform nuclear data investigations. The energy deposited in the silicon layer is lower than what is obtained using reference physics lists (labeled with the specific intra-nuclear cascade model chosen in these predefined classes). The choice of Bertini, BIC (Binary Cascade) or the INCL (Intra-Nuclear Cascade of Liege) does not really impact the energy peak height. The phase-shift solution, at the basis of the Arndt evaluation, provides, instead, some significant discrepancies if used in place of the hadronic elastic model of the CHIPS (CHiral Invariant Phase Space) package used in Geant 4 reference physics lists. Investigations on the most suitable physical models as functions of the incident neutron energy over the whole $20 \mathrm{MeV}-1 \mathrm{GeV}$ domain is ongoing.
A detailed Monte Carlo model of the INFN-PRTs including the reference Arndt data for the only n-p scattering was developed using the Geant4 toolkit [7]. The low statistics (only 25000 entries in coincidence for the first and the second scintillator over $10^{9}$ neutrons simulated) made the computation quite time consuming (about 2 days on a conventional PC in single thread for $10^{9}$ neutrons). Parallel single thread simulations were dispatched on multiple cores to improve statistics and reduce the computing time. Independent seeds were generated using the $\mathrm{C}++11$ std::seed_seq to initialize the Ranecu random number generator in Geant 4 . Figure 3 shows a scatter plot of 512 sim-



Figure 3. Scatter plot of the energy deposition in the first scintillator of the INFN-PRT-L (top). The batch average of $51210^{8}$ neutrons simulations is compared to a single $10^{9}$-neutrons simulation (bottom).

ulations giving the energy deposited in the first scintillator of the INFN-PRT-L detector. $10^{8}$ neutrons at $50 \mathrm{MeV}$ were simulated as primary particles for each run, sampled from a 0.6- $\sigma$-Gaussian spatial distribution. Batch averages and standard deviations were calculated showing a perfect agreement with a single $10^{9}$-neutrons simulation (whose uncertainties were estimated supposing a Poisson distribution). This approach allowed us to perform multiple single-thread stable calculations and rigorous means to quantify uncertainties to be propagated during the particle identification process. Variance reduction techniques are foreseen and their implementation is ongoing. 


\section{Conclusions and Results}

A Polyethylene $\left(\mathrm{C}_{2} \mathrm{H}_{4}\right)$ target was used to maximize the Hydrogen content in a solid target to detect recoil protons from the ${ }^{1} \mathrm{H}(\mathrm{n}, \mathrm{n}){ }^{1} \mathrm{H}$ reaction. In order to extract flux information, a set of measurements, in the original geometrical configuration in figure 1, with Carbon samples of equivalent thicknesses facing the neutron beam, were performed during the experimental campaign.

Using a simplified $\Delta \mathrm{E}$-E detector, the impact of undesired contributions coming from Carbon nuclei in a Polyethylene sample was estimated using Geant $4.10^{9}$ neutrons at

\begin{tabular}{llll}
\hline Ref. Physics List & Any particle & Protons & Deuterons \\
\hline Polyethylene & & & \\
BERT & $76700 \pm 300$ & $76200 \pm 300$ & $450 \pm 20$ \\
BIC & $82600 \pm 300$ & $82100 \pm 300$ & $430 \pm 20$ \\
INCLXX & $85800 \pm 300$ & $83600 \pm 300$ & $1970 \pm 40$ \\
Carbon & & & \\
BERT & $6790 \pm 80$ & $6120 \pm 80$ & $610 \pm 30$ \\
BIC & $13700 \pm 100$ & $13100 \pm 100$ & $530 \pm 20$ \\
INCLXX & $17600 \pm 100$ & $14900 \pm 100$ & $2500 \pm 50$ \\
Hydrogen & & & \\
BERT & $71583 \pm 300$ & $71566 \pm 300$ & $2 \pm 1$ \\
BIC & $71582 \pm 300$ & $71538 \pm 300$ & $19 \pm 4$ \\
INCLXX & $71600 \pm 300$ & $71581 \pm 300$ & $6 \pm 3$ \\
\hline
\end{tabular}

Table 1. Monte Carlo entries in coincidence for a simplified $\Delta \mathrm{E}-\mathrm{E}$ detector, using targets of different materials and several physics lists in Geant4. Pure Carbon and Hydrogen targets are made of fictitious materials having nuclei densities which correspond to those in the Polyethylene target.

$50 \mathrm{MeV}$ impinging on pure Hydrogen and Carbon fictitious samples were simulated using different reference physics lists in Geant4. Nuclei densities were assumed identical to those contained in the Polyethylene target. Table 1 shows how the events in coincidence in the two layers of a simplified $\Delta \mathrm{E}$-E detector are systematically lower than the sum of the events obtained using pure Hydrogen and Carbon, exhibiting self-shielding and attenuation effects. Extensive Monte Carlo simulations have being performed to characterize the INFN-PRT-L and the INFN-PRT-H telescopes. A notable discrimination capability given by the multiple segmentation of the detectors has been preliminarily demonstrated for different neutron energies. Collecting signals in coincidence between several multiple layers allows, in fact, to easily recognize different secondary particles coming from inelastic reactions on Carbon. A fully characterization of the detectors in terms of detection limits, expected punch through energies, intrinsic and global efficiency is ongoing.

Background calculations were performed both to estimate the contributions to the INFN-PRT-L of backscattered secondary particles coming from the target in front of the INFN-PRT-H telescope, and to investigate if particles coming from the former thinner sample may deposit significant energy in the farthest telescope. No significant contributions were observed for both the scenarios.

A thorough comparison between experimental data and Monte Carlo simulations is under achievement. A preliminary result showing Monte Carlo calculations and cali-

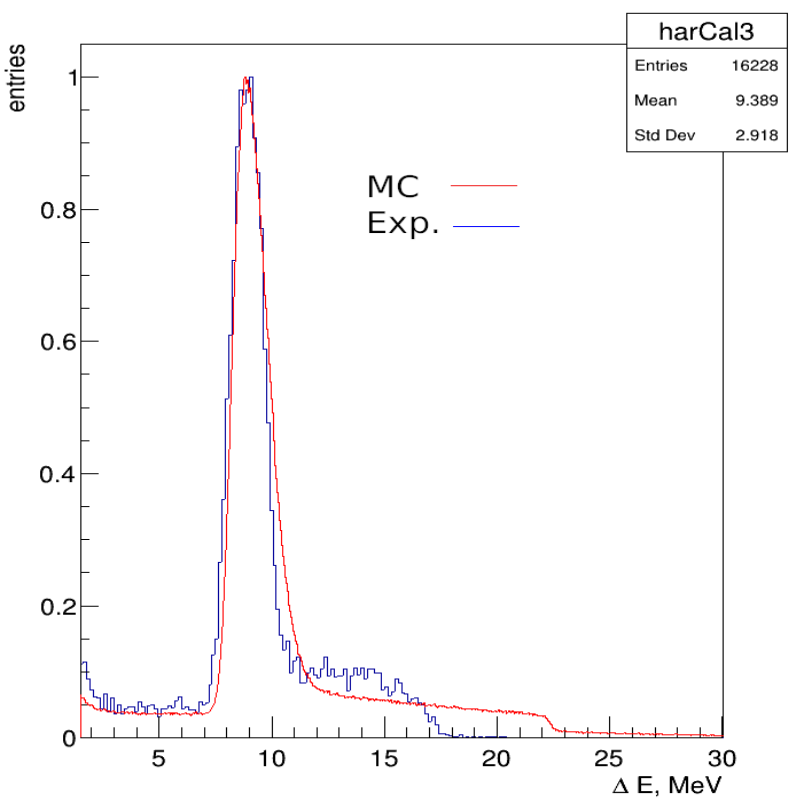

Figure 4. Monte Carlo and experimental data comparison for the energy deposition in the first scintillator of the INFN-PRT-L detector (for $50 \mathrm{MeV}$ neutrons impinging on the target).

brated experimental data for the energy deposited in the first scintillator of the INFN-PRT-L and for $50 \mathrm{MeV}$ neutrons impinging on the target is given in figure 4. Discrepancies can be observed for energies above $11 \mathrm{MeV}$. These may be presumably attributed to deuteron energy depositions coming from reactions on Carbon, which may not be correctly simulated in Geant 4 using the available physics lists.

\section{Acknowledgments}

The authors wish to thank the National Center of the INFN for Research and Development in Information and Communication Technologies (CNAF) for their computational support.

\section{References}

[1] A.D. Carlson et al., Nuclear Data Sheets 148, 143 (2018)

[2] Tarrío et al., Physical Review C 83, 044620 (2011)

[3] A.D. Carlson et al., International Conference on $\mathrm{Nu}-$ clear Data for Science and Technology p. 518 (1991)

[4] R. Nolte et al., Nuclear Science and Engineering 156, 197 (2007)

[5] C. Guerrero et al., European Physical Journal A 49, 27 (2013)

[6] A. Manna et al., International Conference on Nuclear Data for Science and Technology (2019), to be published

[7] S. Agostinelli et al., Nuclear Instruments and Methods in Physics Research Section A: Accelerators, Spectrometers, Detectors and Associated Equipment 506, 250 (2003) 
[8] V. Dangendorf et al., Nuclear Instrumentation Methods A 469, 205 (2001)

[9] R.A. Arndt et al., Physical Review D 35, 128 (1987)
[10] R.A. Arndt et al., Physical Review C 50, 2731 (1994)

[11] IAEA, International evaluation of neutron crosssection standards (2007) 